\title{
Fuzzy logic and its contribution to reliability analysis
}

\author{
H. Furuta \\ Department of Informatics, Kansai University, \\ 2-1-1, Ryozenji-cho, Takatsuki, Osaka 569, Japan
}

In this paper, outline of fuzzy logic and its historical development are first introduced briefly. From the standpoint of fuzzy logic, uncertainties and ambiguities can be classified into several kinds of uncertainties. Here, they are divided into two types of uncertainties; randomness and fuzziness. The reliability analysis based on fuzzy logic is described with emphasis on the probability of fuzzy events and fuzzy probability. Some comments are given on problems that arise in introducing the fuzzy logic in the structural reliability assessment. Several practical examples are presented to demonstrate the applicability of the fuzzy logic in structural engineering. Future problems and possible developments are summarized and discussed.

\section{INTRODUCTION}

Since the pioneer paper by Zadeh in 1965 (Zadeh 1965), fuzzy logic has been developed in various fields. As well as engineering fields, social sciences, medicine, economics, and linguistics have paid attention to the characteristics and advantages of fuzzy logic (Kandel 1991). In Japan, many attempts have been made to develop practical fuzzy controllers in various fields since the pioneer work of the train control of subway in Sendai City (Sugeno 1985). Recently, many Japanese have become familiar with so-called "fuzzy electric appliances" which use micro processors of fuzzy controller to provide flexible and subtle treatment. Especially, washing machine, vacuum cleaner and video camera have gained great success from both technological and commercial points of view.

In the field of civil engineering, many applications have been attempted, some of which have been applied for practical use. In structural engineering, the fuzzy logic has been introduced into the optimum design, reliability and damage assessment, fatigue analysis and cable tension adjustment. Dam control and water quality control are typical examples of applications in hydrology and water resource engineering. In geotechnical engineering, soil or rock classification, seepage analysis, soil improvement and slope stability analysis are promising areas. Traffic control and traffic flow estimation are considered in transportation engineering. In construction engineering, the fuzzy controller for tunneling is famous as a successful application (Furuta 1994a).

In the field of structural engineering, Brown and Leonards (Brown and Leonards 1971) first introduced and discussed the application of fuzzy in 1971, Blockley (Blockley 1975) published an excellent paper on the likelihood of structural accidents. In 1979, Brown 
introduced a fuzzy safety measure combining the application of classic theory of structural reliability with the interpretation of subjective evaluation to obtain more realistic failure rates. Later Shiraishi and Furuta (Shiraishi, Furuta and Kawamura 1982) discussed the application of fuzzy sets to the design of reinforced concrete beams. Furuta et al. (Furuta et. al. 1983) developed the optimum design of earthquake resistant structures using fuzzy mathematical programming. Yao (Yao 1980) and Yao et al. (Yao, Bresler and Hanson 1984) discussed the application of fuzzy sets to safety evaluation of existing structures. Ishizuka et al. (Ishizuka, Fu and Yao 1981) developed a scheme for the combination of evidence using fuzzy sets in their study on expert systems. Chameau et al. (Chameau et. al. 1983) discussed potential applications of fuzzy sets in various subject areas of the civil engineering profession. In 1985, Symposium on Application of Fuzzy Set in Civil Engineering was held at Purdue University, chaired by Brown and Yao (Brown and Yao eds. 1985), and International Symposium on Fuzzy Mathematics in Earthquake Researches was held at Beijing, China (Feng and Liu 1985).

So far, the fuzzy logic has been applied to various structural problems such as damage assessment of existing structures (Furuta et. al. 1991a, Furuta 1993), design planning of bridges (Furuta and Shiraishi 1987), analysis of structural accidents (Blockley 1977, Furuta and Shiraishi 1984), structural reliability assessment (Shiraishi and Furuta 1983, Yao and Furuta 1986), fatigue analysis (Shiraishi, Furuta and Ozaki 1988), and cable tension adjustment (Kaneyoshi et. al. 1990).

In this paper, outline of fuzzy logic and its historical development are first introduced briefly. From the standpoint of fuzzy logic, uncertainties and ambiguities can be classified into several kinds of uncertainties. Here, they are divided into two types of uncertainties; randomness and fuzziness. The reliability analysis based on fuzzy logic is described with emphasis on the probability of fuzzy events and fuzzy probability. Some comments are given on problems that arise in introducing the fuzzy logic in the structural reliability assessment. Several practical examples are presented to demonstrate the applicability of the fuzzy logic in structural engineering. Future problems and possible developments are summarized and discussed.

\section{OUTLINE OF FUZZY LOGIC}

Roughly speaking, fuzzy logic consists of fuzzy set theory and fuzzy measure theory. As well-known, fuzzy set theory was developed by Zadeh, while fuzzy measure was proposed by Sugeno in 1972 (Sugeno 1972). Although the fuzzy sets provides us with an intuitive pleasing method of representing one form of uncertainty, there can be another type of uncertainty which is related to the degree of evidence (Klir and Folger 1988). In order to treat this type of uncertainty, the fuzzy measure was developed.

A fuzzy set is defined as a subset of a universal (sample) space $\Omega$ by a membership function $\mu_{(\mathrm{x})}$ that is a generalization or extension of the characteristic function defining the ordinal sets.

$$
\begin{gathered}
\mu(x) \rightarrow[0,1] \\
C_{A}(x)= \begin{cases}1 & x \in A \\
0 & x \notin A\end{cases}
\end{gathered}
$$


where $C_{A}$ is the characteristic function defined by only two elements, say 0 and 1 , then $A$ is said to be a non-fuzzy or crisp set. Eq. 1 implies that the boundary of the fuzzy set (subset) is not sharp. This property of the fuzzy set is useful in expressing the human subjectivity, because it provides a good interpretation of natural languages which have intrinsically vague or ambiguous and are practical tools for thinking way of human beings.

The arithmetic operations of fuzzy sets commonly used are given as follows:

The membership function for the union of two fuzzy sets $A$ and $B$ is

$$
\mu_{A \cup B}(x)=\max \left\{\mu_{A}(x), \mu_{B}(x)\right\}
$$

On the other hand, the membership function for the intersection of $\mathrm{A}$ and $\mathrm{B}$ is

$$
\mu_{A \cap B}(x)=\min \left\{\mu_{A}(x), \mu_{B}(x)\right\}
$$

The complement of $\mathrm{A}$ is denoted by $\mathrm{A}$, and is given by

$$
\mu_{\bar{A}}(x)=1-\mu_{A}(x)
$$

For two sets $\mathrm{X}$ and $\mathrm{Y}$, a binary relation $\mathrm{R}$ can be considered, which is important for many applications. Using a fuzzy relation $R$, a fuzzy set A is derived from another fuzzy set $B$ as follows:

$$
A=R \circ B
$$

where the symbol $\circ$ denotes the composition of R and B. Eq. 6 can be executed by the extension principle.

$$
\mu_{A}(x)=\max _{y}\left\{\min \left\{\mu_{R}(x, y), \mu_{B}(y)\right\}\right\}
$$

If $\mathrm{R}$ is a fuzzy relation from $\mathrm{X}$ to $\mathrm{Y}$, and $\mathrm{S}$ is a fuzzy relation from $\mathrm{Y}$ to $\mathrm{Z}$, then the composition of $R$ and $S$ is a fuzzy relation which is described with the following membership function:

$$
\mu_{R_{\circ} s}(x, z)=\max _{y}\left\{\min \left\{\mu_{R}(x, y), \mu_{s}(y, z)\right\}\right\}
$$

As mentioned above, the fuzzy measure assigns a value to each crisp sets of the universal set signifying the degree of evidence or belief that a particular element belongs in the set, whereas in the fuzzy set, a value is assigned to each element of the universal set signifying its degree of membership in a particular set with unsharp boundaries.

A fuzzy measure is defined by a function

$$
g: P(X) \rightarrow[0,1]
$$

which assigns to each crisp subset of $X$ a number in the unit interval [0,1]. The axioms of 
fuzzy measures are as follows:

$$
\text { Axiom } 1 \text { (boundary condition) } \quad g(\phi)=0, g(X)=1
$$

Axiom 2 (monotonicity) For every $A, B \in P(X)$, if $A \subseteq B$, then $g(A) \leq g(B)$

Axiom 3 (continuity) For every sequence $A_{i} \in P(X)$ of subsets of X,

$$
\text { if } A_{1} \subseteq A_{2} \subseteq \cdots \cdots \subseteq A_{n} \text {, then } \lim _{n \rightarrow \infty} g\left(A_{n}\right)=g\left(\lim _{n \rightarrow \infty} A_{n}\right)
$$

Then, the probability measure can be derived by replacing the monotonicity condition with the additivity condition:

$$
P(A \cup B)=P(A)+P(B) \quad \text { whenever } A \cap B=\phi
$$

This means that the probability measure is a special case of fuzzy measure, because the additivity axiom is evidently included in the monotonicity axiom.

As representative fuzzy measures, possibility measure, necessity measure and g- $\lambda$ measure are considered. The possibility measure $\Pi$ is defined as follows:

1) $\Pi(\phi)=0, \Pi(X)=1$

2) $\Pi(A \cup B)=\max \{\Pi(A), \Pi(B)\} \quad A, B \in \Omega$

The necessity measure $\mathrm{N}$, which is the dual one of the possibility measure, is as follows:

1)

$$
N(\phi)=0, N(X)=1
$$

2) $\quad N(A \cap B)=\min \{N(A), N(B)\} \quad A, B \in \Omega$

Between these two measure, $\Pi$ and $N$, the following relation holds

$$
N(A)=1-\Pi(\bar{A})
$$

The $g_{\lambda}$ measure is defined as

2)

$$
g_{\lambda}(\phi)=0, g_{\lambda}(X)=1
$$

$$
g_{\lambda}(A \cup B)=\frac{g_{\lambda}(A)+g_{\lambda}(B)-g_{\lambda}(A \cap B)+\lambda g_{\lambda}(A) g_{\lambda}(B)}{1+\lambda g_{\lambda}(A \cap B)}
$$

Using the $g_{\lambda}$ measure, the fuzzy integral (Sugeno 1972) can be defined as follows: 


$$
\int h(x) \circ g_{\lambda}=\max _{\alpha \in 0,1]}\left\{\min \left(\alpha, g_{\lambda}\left(H_{\alpha}(x)\right)\right\}\right.
$$

where $\mathrm{h}(\mathrm{x})$ is the integrand and $\alpha$ is the $\alpha$-level set expressed by

$$
H_{\alpha}(x)=\{x h(x) \geq \alpha\}
$$

The fuzzy integral can provide a useful tool for evaluating the total effect of different kinds of factors.

\section{APPLICATION OF FUZZY LOGIC TO RELIABILITY ANALYSIS}

\subsection{Uncertainties in Civil Engineering}

In spite of the significance success of the probabilistic methods in structural reliability assessment, several investigators have indicated that there are other types of uncertainties in addition to that of randomness (Blockley 1980, Blockley 1983, Brown 1979). It is important and desirable for engineers to recognize several alternative ways of representing uncertainties, and to choose the most appropriate one(s) among them for a particular application (Yao and Furuta 1986).

Here, the application of fuzzy logic to structural reliability is outlined along with probabilistic methods from the viewpoint that a) they are two distinct concepts and b) their applications can be compatible and complementary to each other.

As one of implicit effects of fuzzy logic on structural reliability, one may recognize and distinguish various kinds of uncertainties involved in civil engineering. Generally, the term "uncertainty" may be associated with ambiguity, fuzziness, randomness, vagueness and imprecision of the events under consideration. These uncertainties may be delineated from one another as follows:

(1) Randomness is due to factors in the complex phenomena which are random in nature.

(2) Fuzziness results from the complexity of natural events, the knowledge of which is imprecise and/or incomplete, and/or subjective.

(3) Ambiguity results from the use of natural languages which can be meaningful but not clearly defined.

(4) Blur or vagueness is accompanied with inexact and/or ill-defined figures, pictures and scenes.

(5) Imprecision is due to the lack of information.

For the sake of simplicity, the last four items (i.e., (2) through (5)) are included in the expression of "fuzziness" in contrast to "randomness". Using these two concepts, namely randomness and fuzziness, one can categorize various problems in civil engineering as shown in Fig. 1. In Fig. 1, the upper right-hand corner refers to certain events with neither randomness nor fuzziness. For the analysis of these events, deterministic methods are appropriate in obtaining satisfactory solutions. On the other hand, the lower left-hand comer refers to events which cannot be described with classical mathematical models because of their highly complex and chaotic properties. Now, consider the remaining domain which is not shaded in Fig. 1. Although most common and significant problems in civil engineering belong to this domain, our capability to deal with these problems remains to be studied and developed further.

The probability theory is a useful tool, but it does not cover the whole domain. In the 
application of the probability theory, an event of interest is usually clearly defined. In other words, the application of the probability theory requires us to deal with events which are collections of outcomes of well-defined actions. These events must be subjected to repeatable testing and observations. It is realized that Bayesian statistics serves the purpose of extending the potential application of probabilistic methods. It is, however, noted that the Bayesian approach is not entirely free from the basic probability axioms. To avoid these axioms, Dempster \& Shafer theory (Shafer 1976) has been developed. Including this approach and recognizing the existence of ignorance, the uncertainty diagram can be redrawn as Fig. 2 (Brown and Yao 1985).

Other statistical methods such as regression analysis, discrimination analysis, and multidimensional methods, may also be insufficient to cover all important problems. In any event, it is emphasized here that each method can become more powerful by using it combined with other available methods. The theory of fuzzy logic is one such new approach, with which more meaningful solutions may be obtained for complex problems in the state of nature.

Real-world problems are usually more complex than their corresponding mathematical models. To compensate the gaps between them, some verbal explanation occasionally adds to the results obtained through the models. The concept of fuzzy sets has been developed to deal with the verbal information which is usually meaningful but not clearly defined. Therefore, it is desirable to a) first model actual events with fuzzy sets and b) then analyze them using probability concepts.

To illustrate the need for an additional methodology, several simple examples are presented in the following. Consider an axially loaded plate specimen with butt welds as shown in Fig. 3. If all relevant information (e.g., weld quality, loading and environmental conditions, and statistical quantities) are completely and precisely known, various limit states such as those for ultimate failure yielding and fatigue may be plotted as shown in Fig. 4. In the real world, however, it is difficult to have complete and exact information even for such a simple axially-loaded structural member. For example, the fatigue behavior of butt welds with slag inclusion has been studied experimentally in detail by Bowman et al (Yao and Furuta 1986). Nevertheless, the behavior of actual welded joints depends on many kinds of defects, the effects of which may not be known in a precise manner. As another example along the same line, consider the 1978 AISC (American Institute of Steel Construction) specifications. The allowable stress ranges for various design lives are listed in Table 1. Test data as given by Munse and allowable stresses for 100 000-cycle life are plotted on a Modified Goodman

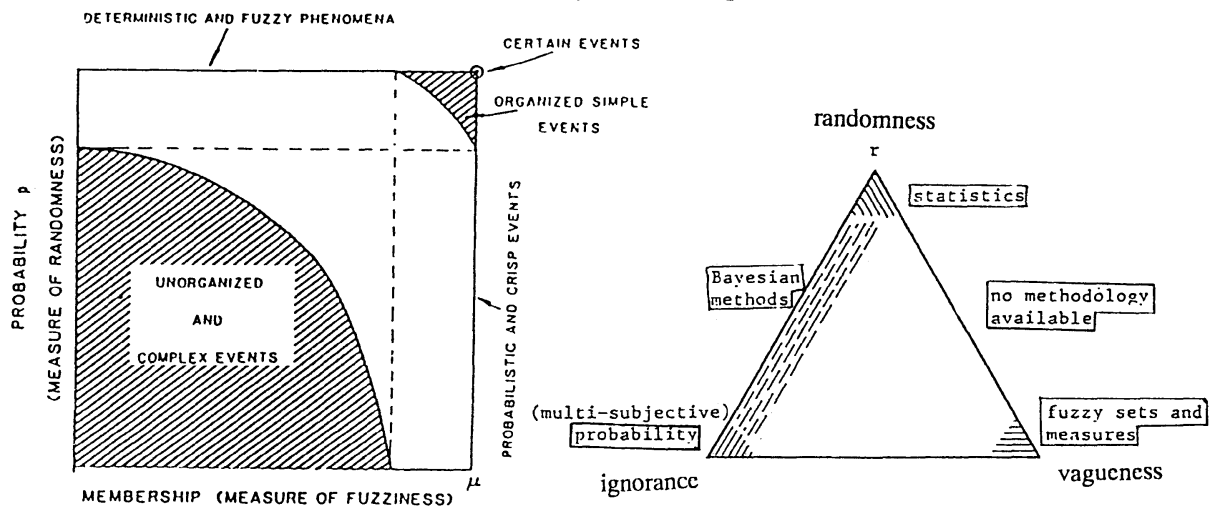

Fig. 1 Probability vs. membership relations.

Fig. 2 Uncertainty diagram 


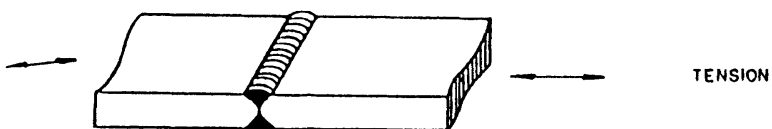

Fig. 3 Axially-loaded and butt-welded plate specimen

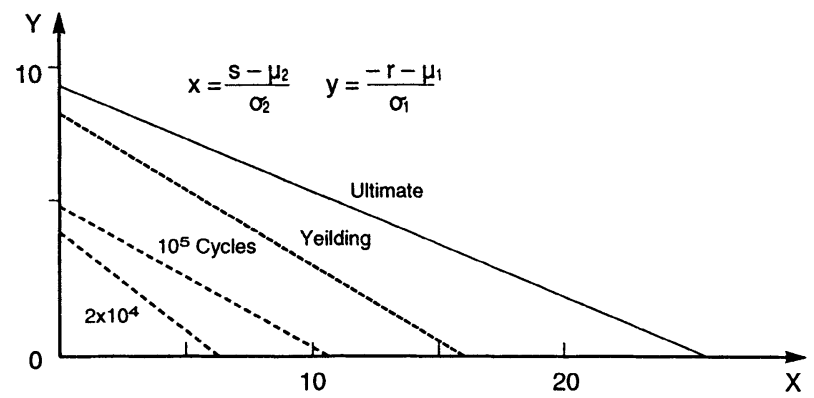

Fig. 4 Limit states for an axially-loaded and butt-welded plate specimen with complete and precise information

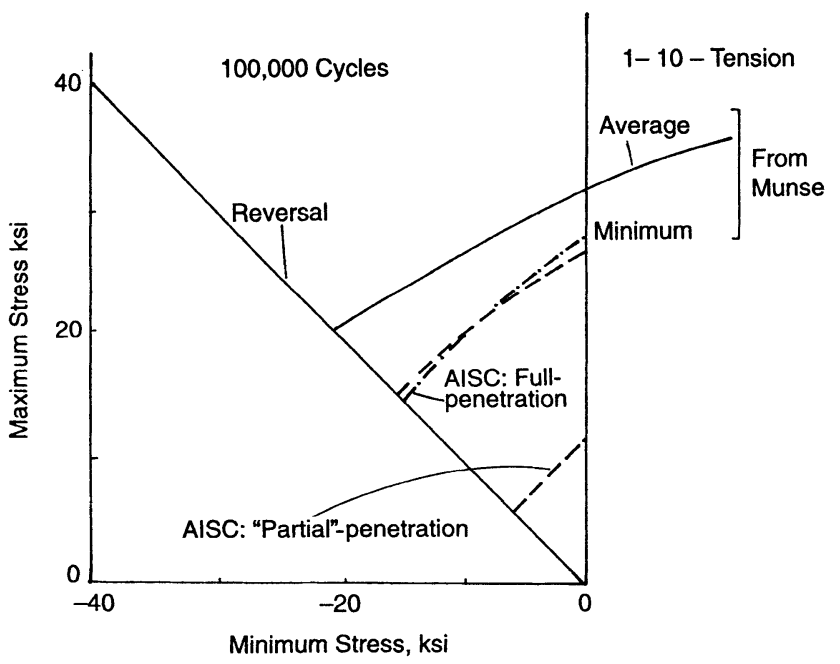

Fig. 5 Modified Goodman diagram for butt welds

Table 1 Allowable fatigue ranges for butt welded joints

\begin{tabular}{lcc}
\hline & \multicolumn{2}{c}{ Allowable stress range, $k s i$} \\
\cline { 2 - 3 } Design life, cycles & Full-penctration & Partial-penctration \\
\hline $20,000-100,000$ & 45 & 15 \\
$100,000-500,000$ & 27.5 & 12 \\
$500,000-2,000,000$ & 18 & 9 \\
$>2,000,000$ & 16 & 8 \\
\hline
\end{tabular}


Diagram as shown in Fig. 5. It is seen that the allowable stresses for "partial penetration of butt welds are much lower than those for "full-penetration" welds. What constitutes "partial" penetration does not seem to be clearly defined.

\subsection{Probability of Fuzzy Events}

Most civil engineering problems involve uncertainties which may be classified into randomness and fuzziness. As an example consider the damage assessment of existing structures. The assessment of structural damage involves several types of uncertainties including randomness in applied loads as well as structural resistances, ambiguity and vagueness involved in observed data or inspection results, imprecision due to the lack of data and limitations in instrumentation, subjective interpretations, and approximation in modeling.

It is possible to evaluate the fuzzy uncertainties with the use of linguistic variables which may be expressed in terms of fuzzy sets. Such uncertainties in the damage assessment are evaluated for each source of available information. As examples, available information sources include (1) building documents, (2) results of a visual examination, (3) field testing, (4) laboratory testing, and (5) structural analysis. By using all available information separately in terms of verbal expressions such as no damage, slight damage, more or less damage, sever damage, and collapse.

Let $\mathrm{E}_{\mathrm{i}}$ denote the evaluation of the information source. $\mathrm{E}_{\mathrm{T}}$ is given by the fuzzy sets with membership functions as given in Fig. 6 and their supports are defined by the real number $[0,1]$. Then, the total assessment may be computed as

$$
E_{T}=\sum_{i=1}^{n} W_{i} E_{i}
$$

where the summation and product correspond to the union and intersection, respectively; $\mathrm{w}_{\mathrm{i}}$ denotes a fuzzy set representing the weight of the i-th evaluation. Because Eq. 17 is an equation of fuzzy quantities, maximum and minimum operators are applied according to the properties of the possibility measure.

$$
\mu_{E T}(u)=\underset{i}{\vee}\left(\mu_{W i}(u) \wedge \mu_{E i}(u)\right)
$$

where $\mu_{E T}, \mu_{W i}$, and $\mu_{E i}$ denote the membership functions of $\mathrm{E}_{\mathrm{T}}, \mathrm{W}_{\mathrm{i}}, \mathrm{E}_{\mathrm{i}}$, respectively. The symbols $\vee$ and $\wedge$ denote maximum and minimum operations, and $u$ is the support for the linguistic variables.

Assume that the damage of the structure is defined by the reserve strength $\mathrm{Z}$ with a probability density function $\mathrm{f}_{\mathrm{Z}}$. Damage occurs when $\mathrm{Z}$ is less than or equal to zero. Then, the probability of having a certain damage state may be calculated as follows:

$$
P_{f}=\int_{-\infty}^{\infty} \mu_{z}(z) f_{z}(z) d z
$$

where the membership function $\mu_{z}$ for the damage state is obtained by the following composition procedure. 


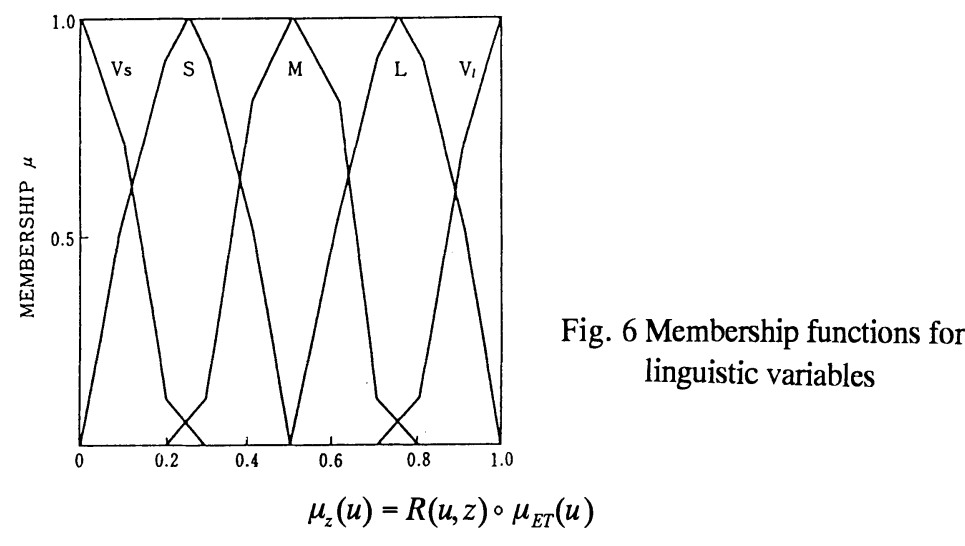

where $\mathrm{R}$ is a fuzzy relation between the total assessment $\mathrm{E}_{\mathrm{T}}$ and the reserve strength $\mathrm{Z}$.

To illustrate this method, consider a numerical example where the probability of having a certain damage state is calculated. The grade of a structural damage is evaluated in the linguistic forms such as "Very large (Vl)", "Large (L)", "Medium (M)", "Small (S)", and "Very small (Vs)", which are characterized by the membership functions as shown in Fig. 6. In Fig. 6, the support $\mathrm{u}$ is a number between zero and one such that $\mathrm{u}=1$ indicates collapse and $\mathrm{u}=0$ indicates no damage. Suppose that the evaluation of Vs, Vl, M, L and S are based respectively on the results of examination of building documents, the visual inspection, the field testing, the laboratory testing, and the structural analysis. Weighing factors are, for the sake of simplicity, assumed to be given by crisp numbers; say 0.3 for building documents, 0.9 for visual inspection, 1.0 for field testing, 0,7 for laboratory testing, and 0.5 for structural analysis. In general, both the linguistic evaluation and weights are determined based on the judgments of experts. Then the total evaluation is calculated as

$$
\begin{aligned}
E_{T}= & 0.3 / 0+0.5 / 0.1+0.5 / 0.2+0.5 / 0.3+0.7 / 0.4 \\
& +1 / 0.5+0.7 / 0.6+0.7 / 0.7+0.7 / 0.8+0.7 / 0.9+0.9 / 1.0
\end{aligned}
$$

Eq. 21 is plotted in Fig. 7. Moreover, assume that the probability density function of reserve strength is given in a discrete form as listed in Table 2 and the fuzzy relation between the total evaluation and the reserve strength is given in Table 3. Using Eq. 21, Table 2 and Table 3, the probability of the damage state is calculated as follows:

$$
\begin{aligned}
P_{f} & =0.9 \times 0.0001+0.5 \times 0.001+0.7 \times 0.005+0.5 \times 0.01+1 \times 0.015 \\
& +0.5 \times 0.02+0.5 \times 0.025+0.5 \times 0.03+0.5 \times 0.04=0.082
\end{aligned}
$$

For the damage state which is defined by $\mathrm{Z}<0$, its occurrence probability is

$$
P_{f}=0.0001+0.001+0.005+0.01+0.015=0.031
$$

Comparing with Eq. 22, the answer as given in Eq. 23 is 2.6 times smaller. 


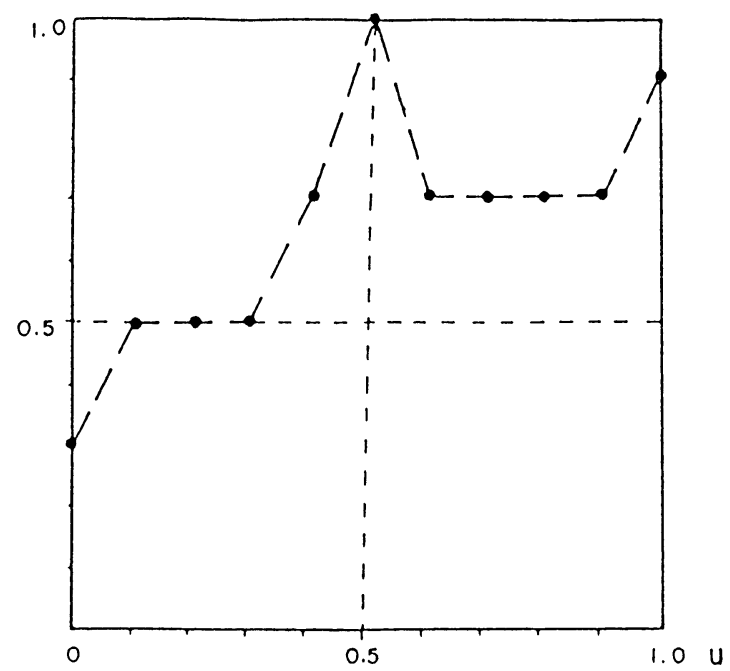

Fig. 7 Membership grades of $\mathrm{E}_{\mathrm{T}}$

Table 2 Probability density function of $\mathrm{Z}$

\begin{tabular}{ll}
\hline Reserve strength (MPa) & Probability \\
\hline-20 & 0.0001 \\
-15 & 0.001 \\
-10 & 0.005 \\
-5 & 0.01 \\
0 & 0.015 \\
5 & 0.020 \\
10 & 0.025 \\
15 & 0.030 \\
20 & 0.04 \\
25 & 0.01 \\
30 & 0.7539 \\
\hline
\end{tabular}

Table 3 Fuzzy relation matrix between $u$ and $z$

\begin{tabular}{lccccccccc}
\hline & -20 & -15 & -10 & -5 & 0 & 5 & 10 & 15 & 20 \\
\hline 0 & & & & & & & & 0.5 & 1.0 \\
0.1 & & & & & & 0.5 & 0.5 & 0.5 & 0.7 \\
0.5 & & & & & & 0.5 & 0.9 & 0.5 & 0.1 \\
0.3 & & & & 0.1 & 0.1 & 0.5 & 0.9 & 0.5 & \\
0.4 & & & & 0.5 & 0.7 & 0.5 & 0.5 & 0.5 & \\
0.5 & & & & 0.5 & 1.0 & 0.5 & & & \\
0.6 & & 0.5 & 0.5 & 0.5 & 0.7 & 0.5 & & & \\
0.7 & & 0.5 & 0.9 & 0.5 & 0.1 & & & & \\
0.8 & 0.1 & 0.5 & 0.9 & 0.5 & & & & & \\
0.9 & 0.7 & 0.5 & 0.5 & 0.5 & & & & & \\
1.0 & 1.0 & 0.5 & & & & & & & \\
\hline
\end{tabular}




\subsection{Fuzzy Probability}

By definition, the probability of fuzzy events is deterministic and crisp number. Meanwhile, there exist situations where the probability is expressed in an ill-defined manner regardless of the fuzziness of the event involved. Such an example can be found in weather forecast. One may say that "the probability of having a cloudy day next Monday is approximately 0.6 " or "it will likely be a cloudy day next Monday". As another example, say that the collapse of a specific bridge is likely to occur within the next 10 years. Or, say that the probability of the crushing of this particular reinforced concrete specimen is approximately 0.5 after a given compressive load is applied.

These ill-defined probabilities are called fuzzy or linguistic probability, which is characterized by a membership function on the probability measure. The fuzzy probability can be mathematically interpreted by considering the upper and lower limits of an integral as fuzzy quantities. By definition, the probability of the occurrence of an event $\mathrm{A}$ is given as

$$
P_{A}=P_{r}[a \leq x \leq b]=\int_{a}^{b} f_{A}(x) d x
$$

where $\mathrm{P}_{\mathrm{r}}\left[{ }^{*}\right]$ is the probability of the event ${ }^{*}$ and $\mathrm{f}_{\mathrm{A}}$ is its probability density function. On the other hand, the fuzzy probability of A can be defined as

$$
P_{A}=P_{r}[\tilde{a} \leq x \leq \tilde{b}]=\int_{\tilde{a}}^{\tilde{b}} f_{A}(x) d x
$$

In Eq. 25, the limits of the integral are fuzzy so that the resultant probability becomes fuzzy. Eq. 25 may be obtained using the extension principle, as will be explained later by an example. Using Eqs. 19 and 25, we can obtain the fuzzy probability of fuzzy events.

$$
P=\int_{\tilde{a}}^{\bar{b}} \mu_{A}(x) f_{A}(x) d x
$$

As an illustrative application of fuzzy probability, consider an example in which the severely damaged state of a uni-axially loaded plate is investigated. Assume that the probability density function of the initial crack length is given by

$$
f_{Y}(y)=\left\{\begin{array}{c}
10(1-5 y) \cdots 0 \leq y \leq 0.2 \\
0 \cdots \cdots \cdots \text { otherwise }
\end{array}\right.
$$

Thus, the severely damaged state is defined when the crack length is between approximately 10 and $20 \%$ of the plate width. Using Eq. 25, the fuzzy probability of such a plate being in severely damaged state is given by

$$
P_{f}=\int_{0 . \overline{1}}^{0 . \tilde{2}} 10(1-5 y) d y
$$

Supposing that fuzzy limits 0.2 and 0.1 are given by 


$$
\begin{aligned}
& 0 . \tilde{2}=0.7 / 0.15+1 / 0.2+0.2 / 0.25 \\
& 0 . \tilde{1}=0.2 / 0.05+1 / 0.1+0.7 / 0.15
\end{aligned}
$$

the calculation of Eq. 28 can be executed by using the extension principle as follows:

$$
\begin{aligned}
P_{f}= & (0.7 \wedge 0.2) /(F(0.15)-F(0.05))+(0.2 \wedge 1) /(F(0.2)-F(0.05)) \\
& +(0.2 \wedge 0.2) /(F(0.25)-F(0.05))+(0.7 \wedge 1) /(F(0.15)-F(0.1)) \\
& +(1 \wedge 1) /(F(0.2)-F(0.1))+(1 \wedge 0.2) /(F(0.25)-F(0.1)) \\
& +(0.7 \wedge 0.7) /(F(0.15)-F(0.15))+(1 \wedge 0.7) /(F(0.2)-F(0.15)) \\
& +(0.2 \wedge 0.7) /(F(0.25)-F(0.15)) \\
= & 0.7 / 0+0.7 / 0.063+0.7 / 0.188+1 / 0.25+0.2 / 0.5+0.2 / 0.563
\end{aligned}
$$

where Eq. 30 are plotted in Fig. 8. Although the fuzzy probability may appeal to our intuition, its calculation is complicated. The fuzzy probability was used in the reliability assessment of damaged structures (Shiraishi and Furuta 1988) and the seismic safety assessment (Furuta et. al. 1991b). However, it is difficult to interpret results of such calculations. In particular, the fuzzy probability of fuzzy events is too broad to be used for solving practical engineering problems at present.

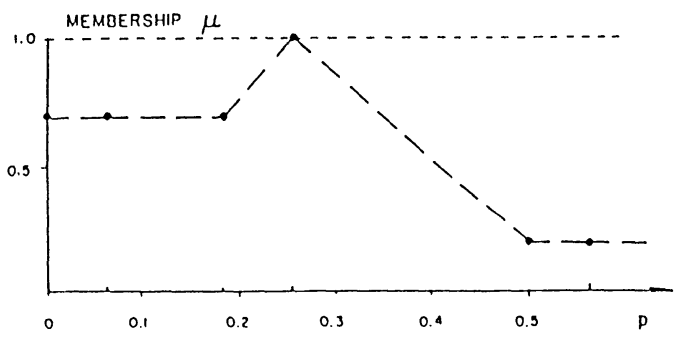

Fig. 8 Membership grades of fuzzy probability

\section{APPLICATION EXAMPLES}

\subsection{Fuzzy Expert System for Damage Assessment}

In order to establish an efficient repair and maintenance program, it is important to evaluate the durability of existing structures. However, the durability assessment of structures is not easy due to the lack of available information and the complex mechanism of structural deterioration. Therefore, the daily maintenance has been so far carried out on the basis of intuition and engineering judgment of experienced engineers. Therefore, attempts have been made to develop rule-based expert systems for assessing the durability of bridge structures.

A number of problems arise when an expert system is built for the practical use. How to treat uncertainties or ambiguities is one of problems which we face occasionally. Here, an expert system is introduced, which has such a remarkable feature that it includes a fuzzy-set manipulation system which can treat fuzzy sets in the process of data handling, rule representation and inference procedure (Furuta et al. 1991a). Using this system, it is 
possible to deal with various kinds of uncertainties and ambiguities involved inherently in the data, rules and inference process in a unified manner. Similar to the usual expert system (Shiraishi, Furuta, Umano \& Kawakami 1987), this durability assessment system consists of interpreter, rule-base and working memory. In order to derive a meaningful conclusion from imprecise and ambiguous information or knowledge, a special inference procedure is necessary. In this system, a fuzzy reasoning method (Zadeh 1975) is employed for this purpose.

In this system, the past records and inspection results are used as the input data. When the inspection results regarding cracks are firstly input into the system, the matching processes for rules concerning their damage cause, damage degree and damage propagation speed are executed to provide a solution for the remaining life.

This system was written in Franz Lisp and implemented on a 32 bit engineering workstation. To make the acquisition of expertise of engineers easier, an attempt was made to apply the technique of neural network for the automatic tuning of fuzzy production rules needed in the durability assessment of RC bridge deck (Furuta et. al. 1993). By introducing the fuzzy-set manipulation system into the expert system, it is possible to utilize the knowledge and rules which are expressed in terms of natural language. This enables us to acquire the expertise with ease. Based on the fuzzy reasoning, it is possible to reduce the number of rules necessary for deriving a meaningful conclusion. The reduction is very useful for building a practical expert system.

\subsection{Fuzzy Fault Tree Analysis}

Up to the present, many bridge failures have been reported, in which various kinds of failure causes were observed. Since bridge structures are large and complicated, it is not easy to identify which failure cause is dominant among the possible causes. Namely, it is difficult to conduct a full-scale experiment or collect satisfactory data to estimate the failure causes or trace the process leading to the failure accident. Difficulty also arises from the fact that the failure accident occurred due to the existence of so-called subjective failure causes such as political, social and economical pressures deriving various kinds of errors.

For the analysis of failure accidents, Fault Tree Analysis (FTA) is a powerful tool and actually has been applied to analyze the accidents of large-scale systems such as highpressure vessels and plant facilities. In FTA, system success or failure can be evaluated by using a so-called structure function, and the probability of success is used as a measure of system reliability. However, this measure may be inconvenient for special problems, one of which is the analysis of bridge accidents. In this problem, our concern is mainly focused on the inter-relationships between every basic event (i. e., failure cause) and its contribution to the underlying bridge accident. It is difficult to judge even whether a basic event occurred or not through the inquiry, because of the complicated mechanism of bridge accident. Then, it may be convenient to define the occurrence of a basic event by a fuzzy set. Namely, the state of a basic event is characterized by a value belonging to [0,1] (grade of membership) instead of $\{0,1\}$. The state of the top event (failure event) can be estimated by using fuzzy minimum and maximum operations.

In order to deal with such subjective failure causes, an attempt was made (Furuta 1994b) to apply fuzzy logic in the analysis of failure accidents of existing bridges.

Although FTA has succeeded in the various kinds of failure analysis, some special problems require an additional improvement on the description of the state of basic events and top event. For problems assessing bridge accidents, an FTA method was developed 
based on fuzzy logic. The method has the following characteristics:

(1) The use of fuzzy minimum and maximum operations enables to simplify the calculation process of structure function.

(2) By using linguistic variables, it is possible to handle the ambiguities involved in the expression of the occurrence of a basic event. In addition, the state of a basic event can be described in a more flexible form, by using the concept of fuzzy logic.

(3) It is possible to discuss the comprehensive safety of bridge by introducing the fuzzy integral into the failure analysis. Using the fuzzy integral, it enables to judge the degree of inevitability of bridge accident in a clear manner. Furthermore, because the fuzzy measure is more flexible than probability measure, the uncertainty and ambiguity involved in the thinking process and way of human beings can be appropriately dealt with.

(4) Considering the comprehensive evaluation value as a weight, it is possible to assess the importance and influence of each failure cause on the bridge accident in a quantitative way.

\section{SUMMARY AND DISCUSSIONS}

In this paper, the outline of fuzzy logic and its contribution to structural reliability assessment were described and discussed. The probability theory is a useful tool, but it does not cover the whole problems in civil engineering. In the application of the probability theory, an event of interest is usually clearly defined. In other words, the application of the probability theory requires us to deal with events which are collections of outcomes of welldefined actions. It is realized that Bayesian statistics serves the purpose of extending the potential application of probabilistic methods. It is, however, noted that the Bayesian approach is not entirely free from the basic probability axioms, and that it requires subject judgments in the determination of apriori distribution and observation or testing data for updating.

Other statistical methods such as regression analysis, discrimination analysis, and multidimensional methods, may also be insufficient to cover all important problems. In any event, it is emphasized here that each method can become more powerful by using it combined with other available methods. The fuzzy logic is one such approach, with which more meaningful solutions may be obtained for complex problems in the state of nature. Real-world problems are usually more complex than their corresponding mathematical models. To compensate the gaps between them, some verbal explanation occasionally adds to the results obtained through the models. The concept of fuzzy sets has been developed to deal with the verbal information which is usually meaningful but not clearly defined.

In this paper, several applications of fuzzy logic in civil engineering are reviewed and discussed from the viewpoint of practical use. Nowadays, the term of "fuzzy" is very popular among Japanese. In Japan, many attempts have been made to apply the fuzzy logic to various engineering problems. In other words, we are in a boom of "fuzzy application". Needles to say, since such boom is easy to run away, we should make clear the essential property and the efficiency of the fuzzy logic.

It may be considered that the advantage of the fuzzy logic lies on the ability of approximation. Introducing the concept of approximation, it is possible to reduce the computation load and time. Furthermore, using the concept of the approximation, it is possible to obtain a practical solution for engineering problems. If the approximate solution is accepted to be satisfactory from practical points of view, it becomes easier to solve complex and large problems of our concern.

One can understand this fact by referring to the damage assessment and reliability 
evaluation of existing structures (Shiraishi and Furuta 1988). When a structure is damaged, its dominant failure modes may change, because the grade, location and cause of damage can greatly affect the system reliability of the structure. However, it is hardly estimated accurately due to the technical and financial constraints, and it may be better to accept a vague or ambiguous evaluation as a practical and meaningful basis for the reliability assessment of the damaged structure. For this purpose, fuzzy sets are useful, because they can represent the verbal expression of experienced engineers in a clear and informative manner.

It should be emphasized again that civil engineers are pursuing the superiority and efficiency of operation, and therefore they will be satisfied when the fuzzy logic can provide us of shortness and save of load and time, though the logical frame of the fuzzy logic is not clear. In fact, the two examples of tunneling machine controller and cable adjustment system have been applied for practical use and succeeded in making the construction time shorter and the operation simpler. Especially, the latter application, e. g., cable tension adjustment is noteworthy because it is only a practical application of fuzzy logic without using the fuzzy control.

Naturally, it is inevitable to recognize the limit of the fuzzy logic. Since we may not always obtain good results for all problems by the fuzzy logic, we should judge which problem is suitable for the application of fuzzy logic. Moreover, we should keep in improving the theoretical basis and proving its validity through practical investigations. Occasionally, the fuzzy logic has been argued on its arbitrariness, say how to determine the membership function, or how to derive the fuzzy relation. It is evident that there is no general answer for these kinds of question. Since appropriate membership functions or relations should be obtained through sufficient observation and testing. In case that observation and testing data can not be collected, knowledge and engineering judgment are important and practical bases for determining them. One example is the optimum design of earthquake resistant structures in which the validity of the membership functions defined by parabolic functions and the minimum operator in aggregating design constraints were examined and proved through the analysis of the questionnaires distributed to experienced engineers. In the fuzzy control for electric appliances or machine operation, the automatic tuning technique has been utilized so as to get better efficiency, based on such new technologies as neural network, chaos and genetic algorithms. The civil engineering problems require the toughness or robustness of solution, because it includes various uncertainties and ambiguities inherently. Therefore, combining these new technologies, the fuzzy logic is expected to provide us of more flexible way of thinking to seek practical and useful solutions.

\section{ACKNOWLEDGMENTS}

The author would like to thank Prof. Naruhito Shiraishi of Kyoto University and Prof. James. T. P. Yao of Texas A\&M University for their valuable advice and continuing encouragement to compile this paper.

\section{REFERENCES}

Blockley, D.(1975).Predicting the likelihood of structural accidents, Proc. ICE, 59, 659-668.

Blockley, D.(1977).Analysis of structural failures, Proc. ICE, 62, 51-74.

Blockley, D.(1980).The Nature of Structural Design and Safety, Ellis Horwood.

Brown, C.(1979).A fuzzy safety measure, ASCE, J Eng. Mech. Div., 105.

Brown, C. and Leonards, R.(1971).Subjective uncertainty analysis, Preprint No.1388, ASCE National 
Structural Meeting, Baltimore.

Brown, C. and Yao, J. eds.(1985).NSF Workshop on Civil Engineering Applications of Fuzzy Sets, W. Lafayette.

Chameau, J. et. al.(1983).Potential applications of fuzzy sets in civil engineering, Int. J. of Man-Machine Stud., 19.

Feng, G. and Liu, X. eds.(1985).Proc. of 1st Int. Sympo. on Fuzzy Mathematics in Earthquake Researches, Beijing.

Furuta, H.(1993).Comprehensive analysis for structural damage based upon fuzzy sets theory, J. of Intelligent and Fuzzy Systems, 1, 55-61.

Furuta, H.(1994a). Application of fuzzy logic in civil engineering in Japan, Proc. of 1st Congress on Computing in Civil Engineering, Washington D.C., 2, 1900-1915.

Furuta, H.(1994b).Analysis of bridge failures using FTA, Proc. of Symposium on Risk Analysis, Ann Arbor.

Furuta, H. et. al.(1983).Optimum aseismic design using fuzzy mathematical programming, IFIP Conference on Computer-Aided Design, Lyon.

Furuta, H. et. al.(1991a).Knowledge-based expert system for damage assessment based on fuzzy reasoning, Computers and Structures, 40, 137-142.

Furuta, H. et. al.(1991b).Seismic reliability analysis of bridge piers using fuzzy probability, Proc. of ICASP5, Mexico, 2, 896-903.

Furuta, H. et. al.(1993).A fuzzy neural system for repairing bridge decks, Proc. of IABSE Colloquium, Beijing.

Furuta, H. and Shiraishi, N.(1984).Fuzzy importance in fault tree analysis, Fuzzy Sets and Systems, 12, 205213.

Furuta, H. and Shiraishi, N.(1987).Bridge design planning based on fuzzy multi-attribute analysis, Preprints of Second IFSA Congress, 1, 337-340160-163.

Ishizuka, M., Fu, K. \& Yao, J.(1981).Inference procedure with uncertainty for problem reduction method, Tech. Rep. CE-STR-81-24, Purdue Univ.

Kandel, A.(1991).Fuzzy Expert Systems, CRC Press.

Kaneyoshi et. al.(1990).Optimum cable tension adjustment using fuzzy regression analysis, Proc. 3rd IFIP WG7.5 Conference, Berkeley.

Klir, G. and Folger, T.(1988).Fuzzy Sets, Uncertainty, and Information, Prentice-Hall.

Shafer, G.(1976).A Mathematical Theory of Evidence, Princeton University Press.

Shiraishi, N., Furuta, H. \& Kawamura, Y(1982).Application of fuzzy set theory to the design of RC beam, Theoretical Applied Mechanics, 31, 173-179.

Shiraishi, N. and Furuta, H.(1983). Reliability analysis based on fuzzy probability, ASCE, J Eng. Mech. Div., 109.

Shiraishi, N., Furuta, H.\& Sugimoto, M.(1985). Integrity assessment of bridge structures based on extended multi-criteria analysis, Proc. of ICOSSAR, 1, 505-509.

Shiraishi, N., Furuta, H., Umano, M. \& Kawakami, K.(1987). An expert system for damage assessment of reinforced concrete bridge deck, Preprints of Second IFSA Congress, 1, 160-163.

Shiraishi, N. and Furuta, H.(1988).System reliability analysis of damaged structures, in D. Frangopol ed., New Directions in Structural System Reliability.

Shiraishi, N., Furuta, H. \& Ozaki, Y.(1988). Application of fuzzy set theory to fatigue analysis of bridge structures, Information Sciences, 45, 175-184.

Sugeno, M.(1972).Fuzzy measure and fuzzy integral, SICE, Japan, 8, 218-226. (in Japanese)

Sugeno, M.(1985).Industrial Applications of Fuzzy Control, North-Holland.

Yao, J.T.P.(1980).Damage assessment of existing structures, ASCE, J Eng. Mech. Div., 106.

Yao, J., Bresler, B. \& Hanson, J.(1984). Condition evaluation and interpretation for existing concrete buildings, Annual Convention of ACI, Phoenix.

Yao, J. and Furuta, H.(1986).Probabilistic treatment of fuzzy events in civil engineering, Prob. Eng. Mech., 1, 1, 58-64.

Zadeh, L. A.(1965). Fuzzy sets, Information and Control, 8, 338-353.

Zadeh, L. A.(1975). The concept of linguistic variable and its application to approximate reasoning - part 2 , Information Science, 8, 43-80. 